\title{
Boundary element analysis of non-planar three-dimensional cracks using complex variables
}

\author{
Dmitry V. Nikolskiy;, Sofia G. Mogilevskaya, Joseph F. Labuz
}

Department of Civil, Environmental, and Geo-Engineering, University of Minnesota, 500 Pillsbury Drive S.E., Minneapolis, MN 55455, USA

\begin{abstract}
This paper reports new developments on the complex variables boundary element approach for solving three-dimensional problems of cracks in elastic media. These developments include implementation of higher order polynomial approximations for the boundary displacement discontinuities and more efficient analytical techniques for evaluation of integrals. The approach employs planar triangular boundary elements and is based on the integral representations written in a local coordinate system of an element. In-plane components of the fields involved in the representations are separated and arranged in certain complex combinations. The Cauchy-Pompeiu formula is used to reduce the integrals over the element to those over its contour and evaluate the latter integrals analytically. The system of linear algebraic equations to find the unknown boundary displacement discontinuities is set up via collocation. Several illustrative numerical examples involving a single (penny-shaped) crack and multiple (semi-cylindrical) cracks are presented.
\end{abstract}

Keywords: Three-dimensional fractures, Boundary element method, Computer simulations

\section{Introduction}

Understanding the mechanisms of initiation and propagation of fractures in rock is of key importance to the mining and petroleum industries. The boundary element method (BEM) is an attractive tool for geomechanical applications as it is capable of efficient treatment of problems featuring large domains containing fractures (described as surfaces of discontinuous displacements) of arbitrary shapes [1,2]. In hydraulic fracturing simulations, the boundary equation for the crack is a fundamental part of a fully coupled model that involves fluid flow [3]. Therefore, accurate and efficient three-dimensional simulators of the fields around multiple non-planar cracks are in great demand.

In our previous publication [4], it was suggested to utilize complex variables for numerical solution of threedimensional crack problems. While some applications of complex variables in three-dimensional elasticity theory have been reported (e.g., [5]-[8]), these publications dealt with the solution of a narrow class of theoretical problems that did not involve numerical simulations. However, in [4] it was shown that complex variables possess some attractive numerical features in simplification of analytical integration and reduction in the number of integral terms, as compared to real variables-based approaches. In the present paper, we extend the complex variables BEM of [4] to incorporate higher order approximations of the displacement discontinuities.

The present complex variables approach is displacement discontinuity (DD)-based in its broader sense (see the literature review in [4] and [9]), as it involves integral representation for tractions in terms of displacement discontinuities on crack surfaces. As in [4], planar triangular elements are employed and in-plane components of tractions, displacement discontinuities, as well as geometric parameters are arranged in various complex combinations. The entries of the matrix of the system of linear algebraic equations, set up via collocation, are calculated analytically by performing analytical integration over the element. However, instead of using simple piecewise constant approximations of the unknowns, as in [4], here we employ polynomials up to second order. In addition,

\footnotetext{
${ }^{*}$ Corresponding author. Tel.: +1-612-625-4579; fax: +1-612-626-7750. E-mail address: nikol047@umn.edu (D.V. Nikolskiy).
} 
instead of using an iterative integration procedure, which does not fully utilize the advantages of complex variables, we use the integration technique reported in [10]. The technique is based on representations for complex functions that reduce the integrals over the area of the element to those over its contour. We show that all entries of the influence matrix can be composed of various combinations of one generic integral, its derivatives, and their complex conjugates. The same integrals and combinations are involved in the representations of the elastic fields everywhere in the computational domain. The analytical expression for the generic integral over a triangular element is provided.

The goal of the present paper is to study the influence of higher order approximations on the accuracy of evaluation of the fields. While we do not introduce special crack tip shape functions for DD (as we are aiming at the applications, e.g. hydraulic fracturing, that do not necessarily exhibit square root asymptotic behavior), we show that higher order approximations still allow for capturing stress asymptotic behavior near the tips of dry cracks. The approach is tested on several examples, namely (i) the problem of a penny shaped crack under normal and shear far-field load, and (ii) the problem of two semi-cylindrical coaxial cracks under biaxial far-field load. Some of the results are tabulated to serve as benchmarks for future investigations.

\section{Problem formulation and basic equations}

We are concerned with the BEM solution of the problem of the stress state in an infinite elastic domain containing non-planar cracks of arbitrary shapes and subjected to far-field stresses (zero body force is assumed). The sign convention is that positive tensile stress.

BEM employs integral representations, which are equivalent to the governing partial differential equations of a specific problem, but expresses the solution of that problem in terms of integrals over the boundary of the domain of interest. The representation for the tractions $\mathbf{t}(\mathbf{x})$ at some point $\mathbf{x}$ located outside of the crack surfaces on the plane characterized by normal vector $\mathbf{n}(\mathbf{x})$ has the following form:

$$
t_{j}(\mathbf{x})=-\int_{\Sigma} H_{j k}(\mathbf{x}, \boldsymbol{\xi}) \triangle u_{k}(\boldsymbol{\xi}) d \Sigma_{\xi}
$$

where $\Sigma$ is the totality of cracks' surfaces, $\boldsymbol{\xi} \in \Sigma, \triangle u_{k}$ are the components of the displacement discontinuity (DD) $\boldsymbol{\Delta} \mathbf{u}=\mathbf{u}^{+}-\mathbf{u}^{-}$, in which $\mathbf{u}(\mathbf{x})$ is the displacement vector with the components $u_{k}(k=1 \ldots 3)$, " + and " - " identify the displacements on the opposite sides of the crack, $t_{j}$ are the components of the traction vector. The direction of the normal for the crack may be chosen arbitrarily. In the notations used, this direction indicates the outward normal for the " + " side (inward normal for the " - " side) of the crack.

Another notation adopted in Eq. (1) is related to the tensor $\mathbf{H}$ that represents the kernel of the hypersingular potential associated with Kelvin's fundamental solution [9], [11]-[13]. Its components $H_{j k}(\mathbf{x}, \boldsymbol{\xi})$ are given by the following expression:

$$
\begin{aligned}
H_{j k}(\mathbf{x}, \boldsymbol{\xi}) & =\frac{\mu}{4 \pi(1-\nu)}\left\{\frac{1-2 \nu}{r^{3}}\left[n_{m}(\mathbf{x}) n_{m}(\xi) \delta_{j k}+n_{j}(\xi) n_{k}(\mathbf{x})\right]-\frac{1-4 \nu}{r^{3}} n_{j}(\mathbf{x}) n_{k}(\xi)+\right. \\
& +\frac{3(1-2 \nu)}{r^{5}}\left[r_{m} n_{m}(\xi) n_{j}(\mathbf{x}) r_{k}+r_{m} n_{m}(\mathbf{x}) r_{j} n_{k}(\xi)\right]+\frac{3 \nu}{r^{5}} r_{l} n_{l}(\xi)\left[r_{m} n_{m}(\mathbf{x}) \delta_{j k}+\right. \\
& \left.\left.+r_{j} n_{k}(\mathbf{x})\right]+\frac{3 \nu}{r^{5}} r_{k}\left[n_{m}(\mathbf{x}) n_{m}(\xi) r_{j}+r_{m} n_{m}(\mathbf{x}) n_{j}(\xi)\right]-\frac{15}{r^{7}} r_{m} n_{m}(\mathbf{x}) r_{l} n_{l}(\xi) r_{j} r_{k}\right\}
\end{aligned}
$$

where $\delta_{j k}$ is Kronecker's symbol, $r_{k}=x_{k}-\xi_{k}, r=|\mathbf{x}-\boldsymbol{\xi}|, n_{k}(\mathbf{x})$ are the components of $\mathbf{n}(\mathbf{x}), n_{k}(\boldsymbol{\xi})$ are the components of the outward unit normal vector $\mathbf{n}(\boldsymbol{\xi})$ at the point $\boldsymbol{\xi}, \mu$ is the shear modulus, and $\nu$ is the Poisson's ratio.

The case of $\mathbf{x} \in \Sigma$ is handled as the limiting case in which the point $\mathbf{x}$ is allowed to reach the crack surface. Note that for the limit of the integral in Eq. (1) to exist, certain smoothness conditions are required for the boundary and the approximating functions for $\Delta \mathbf{u}\left(\Delta \mathbf{u}(\boldsymbol{\xi}) \in C^{1, \alpha}[2,13]\right)$.

We assume that the tractions on $\Sigma$ are prescribed. In case of far-field load, a standard superposition procedure is adopted, in which the problem is decomposed into two problems: one of an infinite domain (without cracks) 
under the far-field load and another one of an infinite domain containing the crack loaded by the prescribed corrective tractions and zero far-field stress. To find the approximate solution of the problem, the boundary is discretized, approximating (shape) functions for the unknowns are introduced, the integrals associated with each degree of freedom (and the corresponding shape function) are evaluated, and the system of linear algebraic equations is set by using the limit to the boundary and matching the prescribed boundary data (the standard steps of the BEM procedure). After the solution of the system of algebraic equations is found, the stresses inside the domain $\Omega$ can be reconstructed using the superposition of the prescribed far-field stress with the stresses given by the following discretized analog of Eq. (1) with appropriately chosen normal vectors $\mathbf{n}(\mathbf{x})$ :

$$
t_{j}(\mathbf{x})=-\sum_{s=1}^{N_{e}} \int_{E_{s}} H_{j k}(\mathbf{x}, \boldsymbol{\xi}) \triangle u_{k}(\boldsymbol{\xi}) d \Sigma_{\xi}
$$

The integrals involved in (3) are regular integrals and evaluated using the same procedures as the ones used to assemble the matrix of the system of algebraic equations (influence matrix).

The displacements at the point $\mathbf{x}$ inside the domain of interest can be obtained (up to a rigid body movement) from the discretized analog of the following equation:

$$
u_{j}(\mathbf{x})=-\sum_{s=1}^{N_{e}} \int_{E_{s}} T_{j k}(\mathbf{x}, \boldsymbol{\xi}) \triangle u_{k}(\boldsymbol{\xi}) d \Sigma_{\xi}
$$

where the components $T_{j k}$ of the tensor $\mathbf{T}$ that represents the kernel of the double layer potential are given in [4]. It should be noted that the formulation presented can be extended to the case of a finite domain containing cracks by including in Eqs. (1), (3)-(4) the corresponding integrals over the boundary of the finite domain (see $[4])$.

\section{Assumptions and complex notations for the fields and geometry}

As noted, in the present work planar triangular elements are used to discretize the cracks surfaces. The unknown displacement discontinuities on each element $E_{s}$ are approximated by the linear combination of quadratic Lagrange polynomials associated with nodal points (6 per element). The positions of these points may vary; in the present paper two arrangements are studied: a) nodes located on the contour of the element (including vertices) and $\mathbf{b}$ ) nodes located inside the element. The case a) provides continuity of DDs across the edges, which the case $\mathbf{b}$ ) does

not. Note that the collocation points can only be located inside the element due to the smoothness conditions required for the boundary and the approximating functions.

The local Cartesian coordinate system is introduced on the element $E_{s}$ as in [4]: $\widehat{\mathbf{e}}_{1} \widehat{\mathbf{e}}_{2} \widehat{\mathbf{e}}_{3}$ is the right-handed orthonormal basis where $\widehat{\mathbf{e}}_{1}$ is parallel to the edge between two vertices of the element, $\widehat{\mathbf{e}}_{3}=-\mathbf{n}(\boldsymbol{\xi})$, and $\widehat{\mathbf{e}}_{2}=$ $\widehat{\mathbf{e}}_{3} \times \widehat{\mathbf{e}}_{1}$. The origin of this coordinate system may be chosen arbitrarily. From now on, components of vectors and tensors are given in the local coordinate system of $E_{s}$ (see Fig. 1).

The following combinations associated with the vector $\mathbf{r}$ that connects the point $\xi \in E_{s}$ and the field point $\mathbf{x}=\left(x_{1}, x_{2}, x_{3}\right)$ are introduced:

$$
z=x_{1}+i x_{2} ; \quad \tau=\xi_{1}+i \xi_{2} ; \quad h=\xi_{3}-x_{3}
$$

where $i^{2}=-1$. With notations (5) the distance $r$ can be expressed as

$$
r=\sqrt{(\tau-z)(\bar{\tau}-\bar{z})+h^{2}}
$$

where the bar over the symbol defines the complex conjugate of $z$, i.e. $\bar{z}=x_{1}-i x_{2}$.

For the normal vector $\mathbf{n}(\mathbf{x})$ to the plane containing the point $\mathbf{x}$, we introduce the following notations:

$$
\underline{n}(\mathbf{x})=n_{1}(\mathbf{x})+i n_{2}(\mathbf{x}) ; \quad n_{\mathbf{n}}=n_{3}
$$




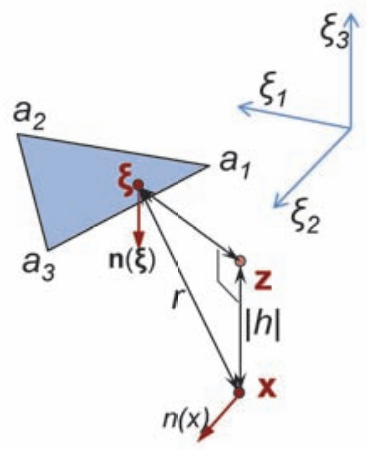

Figure 1: Boundary element. Local coordinate system and notations.

We also introduce the combinations of similar kind for traction and displacement components:

$$
\begin{aligned}
\underline{t} & =t_{1}+i t_{2} ; \quad t_{\mathbf{n}}=t_{3} \\
\underline{u} & =\Delta u_{1}+i \Delta u_{2} ; \quad u_{\mathbf{n}}=\Delta u_{3}
\end{aligned}
$$

With these notations, a typical quadratic shape function can be expressed as

$$
A^{(N)}(\tau, \bar{\tau})=\sum_{\substack{m, n=0 \\ m+n \leq 2}}^{2} \kappa_{m n}^{(N)}(\tau-z)^{m}(\bar{\tau}-\bar{z})^{n}
$$

where $\tau$ and $\bar{\tau}$ are understood as independent variables, the superscript $N=1 \ldots 6$ identifies the node, and the coefficients $\kappa_{m n}^{(N)}=\overline{\kappa_{n m}^{(N)}}$ are defined by the arrangement of the nodes on the element $E_{s}$.

For example, for the real function given by the following equation:

$$
A\left(x_{1}, x_{2}\right)=1-3 x_{1}-3 x_{2}+2 x_{1}^{2}+2 x_{2}^{2}+4 x_{1} x_{2}
$$

one gets

$$
\kappa_{00}=1, \kappa_{10}=\frac{3}{2}(i-1), \kappa_{01}=-\frac{3}{2}(i+1), \kappa_{20}=-i, \kappa_{02}=i, \kappa_{11}=2
$$

\section{Structure of the integrals}

It can be shown that, for polynomial approximations of the unknowns, Eq. (3) written for a single element $E_{s}$ is composed of the integrals of the following type:

$$
I_{m, n, k}=\overline{I_{n, m, k}}=\int_{E_{s}} \frac{(\tau-z)^{m}(\bar{\tau}-\bar{z})^{n}}{r^{k}} d \Sigma_{\xi}, k=3,5,7
$$

In particular, after substituting the shape function of Eq. (9) into the part of Eq. (1) associated with $E_{s}$, one gets 


$$
\begin{aligned}
& \underline{t}^{(1)}(\mathbf{x})=\sigma_{13}(\mathbf{x})+i \sigma_{23}(\mathbf{x})= \\
& \frac{\mu}{8 \pi(1-\nu)} \sum_{N=1}^{6} \sum_{m, n=0}^{2} \kappa_{m n}^{(N)}\left[\underline{u}^{(N)}\left((\nu-2) I_{m, n, 3}-3 \nu h^{2} I_{m, n, 5}-15 h^{4} I_{m, n, 7}\right)\right. \\
& +\bar{u}^{(N)}\left(-3 \nu I_{2+m, n, 5}+15 h^{2} I_{2+m, n, 7}\right) \\
& \left.+u_{\mathbf{n}}^{(N)}\left(-6 h I_{1+m, n, 5}+30 h^{3} I_{1+m, n, 7}\right)\right] \\
& \underline{t}^{(2)}(\mathbf{x})=\sigma_{11}(\mathbf{x})-\sigma_{22}(\mathbf{x})+2 i \sigma_{12}(\mathbf{x})= \\
& \frac{\mu}{8 \pi(1-\nu)} \sum_{N=1}^{6} \sum_{\substack{m, n=0 \\
m+n \leq 2}}^{2} \kappa_{m n}^{(N)}\left[\underline{u}^{(N)}\left(-12 \nu h I_{1+m, n, 5}+15 h I_{2+m, 1+n, 7}\right)\right. \\
& +\bar{u}^{(N)}\left(15 h I_{3+m, n, 7}\right) \\
& \left.+u_{\mathbf{n}}^{(N)}\left(6(2 \nu-1) I_{2+m, n, 5}+30 h^{2} I_{2+m, n, 7}\right)\right] \\
& t^{(3)}(\mathbf{x})=\sigma_{11}(\mathbf{x})+\sigma_{22}(\mathbf{x})= \\
& \frac{\mu}{4 \pi(1-\nu)} \sum_{N=1}^{6} \sum_{m, n=0}^{2} \kappa_{m n}^{(N)}\left[\underline{u}^{(N)}\left(6(\nu-1) h I_{m, 1+n, 5}+15 h I_{1+m, 2+n, 7}\right)\right. \\
& +\bar{u}^{(N)}\left(6(\nu-1) h I_{1+m, n, 5}+15 h I_{2+m, 1+n, 7}\right) \\
& \left.+u_{\mathbf{n}}^{(N)}\left(-(2 \nu+1) I_{m, n, 3}+6(\nu+2) h^{2} I_{m, n, 5}-15 h^{4} I_{m, n, 7}\right)\right] \\
& t^{(4)}(\mathbf{x})=\sigma_{33}(\mathbf{x})= \\
& \frac{\mu}{4 \pi(1-\nu)} \sum_{N=1}^{6} \sum_{m, n=0}^{2} \kappa_{m n}^{(N)}\left[\underline{u}^{(N)}\left(-3 h I_{m, 1+n, 5}+15 h^{3} I_{m, 1+n, 7}\right)\right. \\
& m+n \leq 2 \\
& +\bar{u}^{(N)}\left(-3 h I_{1+m, n, 5}+15 h^{3} I_{1+m, n, 7}\right) \\
& \left.+u_{\mathbf{n}}^{(N)}\left(-I_{m, n, 3}-6 h^{2} I_{m, n, 5}+15 h^{4} I_{m, n, 7}\right)\right]
\end{aligned}
$$

Integrals of Eq. (10) can be obtained from the following generic integral

$$
I_{m n}=I_{m, n, 1}=\int_{E_{s}} \frac{(\tau-z)^{m}(\bar{\tau}-\bar{z})^{n}}{r} d \Sigma_{\xi}
$$

by differentiation over $h^{2}$ : 


$$
\begin{aligned}
I_{m, n, 3} & =-2 \frac{\partial}{\partial\left(h^{2}\right)} I_{m n} \\
I_{m, n, 5} & =\frac{4}{3} \frac{\partial^{2}}{\partial\left(h^{2}\right)^{2}} I_{m n} \\
I_{m, n, 7} & =-\frac{8}{15} \frac{\partial^{3}}{\partial\left(h^{2}\right)^{3}} I_{m n}
\end{aligned}
$$

The evaluation of generic integral (15) was described in [10] for elements bounded by straight lines and circular arcs; for convenience, we summarize the corresponding results for triangular elements in Appendix A.

\section{$5 \quad$ Evaluation of boundary tractions}

The expressions for $t^{(1)}(\mathbf{x})-t^{(4)}(\mathbf{x})$ of Eqs. (11)-(14) represent the case when the point $\mathbf{x}$ is located outside of the crack surface. To meet the prescribed tractions on that surface and obtain the unknown displacement discontinuities one has to perform a limiting procedure in which the point $\mathbf{x}$ is allowed to reach some point on the surface. This procedure is, in fact, straightforward, except for the case when this point belongs to the element or is located on the plane that contains it; in this case, the limits of tractions can be taken as if $\mathbf{x} \rightarrow \mathbf{z}$ or $h \rightarrow 0$. As mentioned in Section 3, $\mathbf{z}$ must be an internal point of the element. Although some particular integrals involved in Eqs. (11)-(14) may diverge when $h \rightarrow 0$, the divergent terms cancel out after the assembly leading to continuous final closed-form expressions for $t^{(1)}(\mathbf{z})-t^{(4)}(\mathbf{z})$. These limit expressions are as follows:

$$
\begin{aligned}
& t^{(1)}(\mathbf{z})=\sum_{N=1}^{6}\left[\frac{(2-\nu)}{4} U^{(N)} \underline{u}^{(N)}+\frac{\nu}{2} V^{(N)} \bar{u}^{(N)}\right] \\
& t^{(2)}(\mathbf{z})=\sum_{N=1}^{6}(1-2 \nu) V^{(N)} u_{\mathbf{n}}^{(N)} \\
& t^{(3)}(\mathbf{z})=\sum_{N=1}^{6} \frac{1+2 \nu}{2} U^{(N)} u_{\mathbf{n}}^{(N)} \\
& t^{(4)}(\mathbf{z})=\sum_{N=1}^{6} U^{(N)} u_{\mathbf{n}}^{(N)}
\end{aligned}
$$

where

$$
\begin{aligned}
U^{(N)}=\sum_{m=1}^{3} & \left\{\kappa_{00}^{(N)} \frac{\sin \chi_{m n}}{\left|d_{m}\right|}+L_{m n}\left(2 \operatorname{Re}\left(\kappa_{10}^{(N)} c_{m}\right)-\kappa_{11}^{(N)}\left|d_{m}\right|\right)\right. \\
& \left.+2\left|d_{m}\right| \operatorname{Re}\left(\kappa_{20}^{(N)} c_{m}^{2}\left[L_{m n}+2 i \exp \left(i \chi_{m n}\right)\right]\right)\right\}\left.\right|_{n=m} ^{m+1} \\
V^{(N)}=\sum_{m=1}^{3} & \left\{\kappa_{00}^{(N)} \frac{c_{m}^{2}}{2\left|d_{m}\right|}\left[\sin \chi_{m n}-2 i \exp \left(i \chi_{m n}\right) \cos \chi_{m n}\right]\right. \\
& -\kappa_{10}^{(N)} \frac{c_{m}^{3}}{8}\left[4 L_{m n}+i \exp \left(i \chi_{m n}\right)\left(\exp \left(2 i \chi_{m n}\right)+8\right)\right] \\
& +\kappa_{01}^{(N)} \frac{c_{m}}{8}\left[12 L_{m n}+5 i \exp \left(i \chi_{m n}\right)\right] \\
& -\kappa_{20}^{(N)} \frac{\left|d_{m}\right| c_{m}^{4}}{2}\left[3 L_{m n}-2 i \exp \left(i \chi_{m n}\right)\left(\exp \left(2 i \chi_{m n}\right)-3\right)\right] \\
& \left.-\kappa_{02}^{(N)} \frac{3\left|d_{m}\right|}{2} L_{m n}+\kappa_{11}^{(N)} \frac{3\left|d_{m}\right| c_{m}^{2}}{2}\left[L_{m n}+2 i \exp \left(i \chi_{m n}\right)\right]\right\}\left.\right|_{n=m} ^{m+1}
\end{aligned}
$$


and

$$
\begin{aligned}
L_{m n} & =\tanh ^{-1}\left(\sin \chi_{m n}\right) \\
& =\left(\log \left(1+\sin \chi_{m n}\right)-\log \left(1-\sin \chi_{m n}\right)\right) / 2 \\
\chi_{m n} & =\arg \left(a_{n}\right)-\arg \left(d_{m}\right) \\
c_{m} & =\frac{d_{m}}{\left|d_{m}\right|}
\end{aligned}
$$

The notations $a_{n}, d_{m}, \chi_{m n}$ are explained in Fig. 13 of Appendix A.

\section{Assembly and solution of the system of algebraic equations}

The system of linear algebraic equations is set by using a standard collocation method. The boundary tractions at each collocation point are represented as a sum of the contributions due to all boundary elements (which, in turn, are composed of the contributions due to all degrees of freedom associated with those elements) and matched with the prescribed boundary data at that point. Before the summation, the tractions of Eqs. (11)-(14) or (17) are transformed from the coordinate system of the analyzed element to the global coordinate system (using the coordinate transformation determined by the orientation of the element [4]).

We adopt the algorithm in which the numbers of nodes and collocation points are equal, so each collocation point is somewhat associated with a certain node. The results reported in the next Sections have been obtained with the following arrangements of nodal and collocation points:

a) continuous elements: nodal points coincide with the mesh vertices; additional nodes are placed on the edges. No nodes are placed at the vertices located on the crack front curve where zero DD conditions are enforced (that means that the corresponding rows and columns are excluded from the influence matrix). The collocation point associated with a specific node is located inside the element with the largest area among those sharing that node and placed at $1 / 4-1 / 3$ of the distance between the node and the element centroid. Note that such an arrangement may result in some elements containing no collocation points.

b) discontinuous elements: nodal points coincide with collocation points and are located inside the element at $1 / 4-1 / 3$ of the distances between the vertices and the element centroid.

An example of a mesh (a penny-shaped crack discretized with 40 elements) and arrangements of nodal and collocation points associated with a part of the mesh (a rectangle identified by the dashed line) are shown in Fig. 2 for both arrangement schemes $\mathbf{a}$ and $\mathbf{b}$.

We emphasize that while the approximations adopted in the scheme a are continuous, they are only sufficiently smooth inside each element but can lack the required smoothness $\left(\Delta \mathbf{u}(\boldsymbol{\xi}) \in C^{1, \alpha}\right)$ between the elements. Thus, in this scheme, the collocation points can be placed only inside the elements.

As we stated, we did not employ special tip shape functions. However, the second order approximations for the DD may allow us to capture the asymptotic behavior of stress field near the crack tips, which constant approximations do not. To further facilitate this feature, some nodes of the tip elements can be shifted towards the tip (see Fig. 2) in somewhat similar manner as in the so-called "quarter-point elements" used in Finite Element crack analysis.

The system of equations is solved via either direct Gaussian or iterative methods (the MATLAB package and its built-in procedures are used) to obtain the unknown displacement discontinuities at the nodal points. No fast solver is implemented at this point; it will be a subject of future work.

\section{Evaluation of the stresses inside the computational domain}

The components of the stress tensor at any arbitrary point inside the domain of interest can be calculated by substituting the obtained displacement discontinuities $\underline{u}^{(N)}$ and $u_{\mathbf{n}}^{(N)}$ on each element into Eqs. (11)-(14) or (17) 


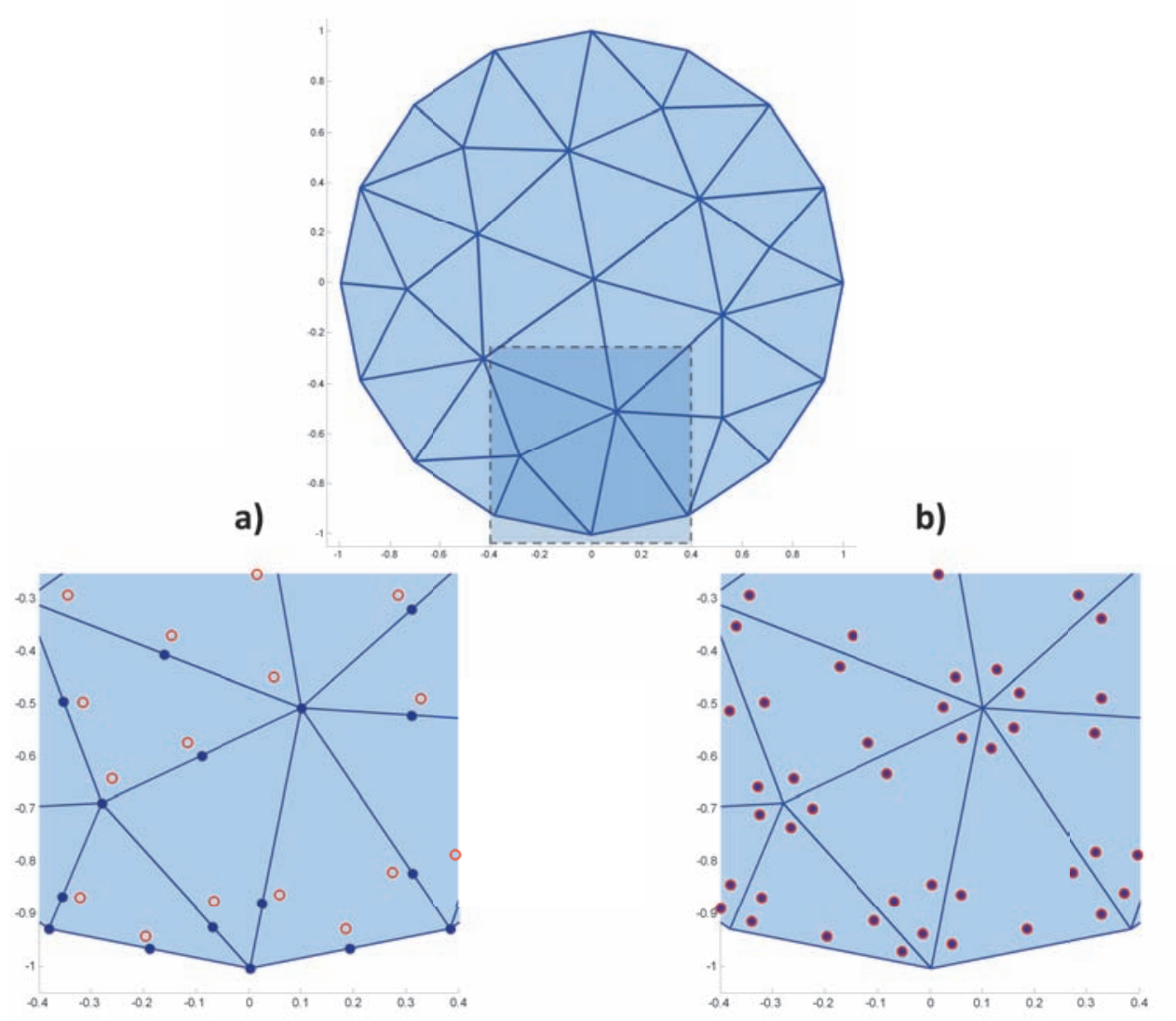

Figure 2: Example of the boundary mesh (penny-shaped crack) and two schemes of nodes arrangement: a) continuous DD b) discontinuous DD.

with appropriately chosen normal vectors $\mathbf{n}(\mathbf{x})$ and combining the contributions of all elements (the corresponding coordinate transformations should be used in this procedure). Note that, since the "limit after discretization" approach is employed, the integrals involved in Eqs. (11)-(14) and (17) are evaluated using the same algorithm as the one employed in the assembly of the system of algebraic equations.

\section{Benchmark 1: Penny-shaped crack}

We start with the well-known and well studied ([8],[14]-[17]) problem of a penny-shaped crack in an infinite domain under normal or shear load. Its known analytical solutions for the DDs and for the stresses around the crack have been briefly summarized in the Appendix of [4] and will not be repeated here. In [4], we also reported the numerical solution of this problem with constant approximation of unknowns. Here, we will compare those results with the new results obtained with the use of quadratic approximations. In the following we assume that the Poisson's ratio of the medium is $\nu=0.25$ and the penny-shaped crack is of unit radius.

Fig. 3 compares the dimensionless displacement discontinuities $\Delta \mathbf{u} / R S\left(S=\sigma_{z z}^{\infty} / \mu\right.$ for normal load and $S=\sigma_{x z}^{\infty} / \mu$ for shear load) along the crack surface obtained using the two schemes: continuous quadratic approximations (scheme a with 121 elements) and discontinuous quadratic approximations (scheme b with 40 elements). The schemes possess roughly the same number of degrees of freedom (243 and 240 collocation points, respectively). The reference analytical solution is identified by the solid line. One can see that the scheme a provides better results for both normal and shear loads. We believe that it is due to the fact that in this scheme the zero DD conditions were enforced at vertices on the crack front.

Fig. 4 compares the results for the dimensionless displacement discontinuities (both for normal and shear 
loads) obtained with continuous quadratic approximations and with constant approximations. The reference analytical solution is again identified by the solid line. It could be seen that, for roughly the same numbers of degrees of freedom, continuous quadratic approximation scheme yields much better accuracy, especially in the crack tip region.

Fig. 5 demonstrates the mesh convergence of the method by comparing the results for the dimensionless displacement discontinuities (for both normal and shear loads) obtained on meshes with 121 and 1025 elements.
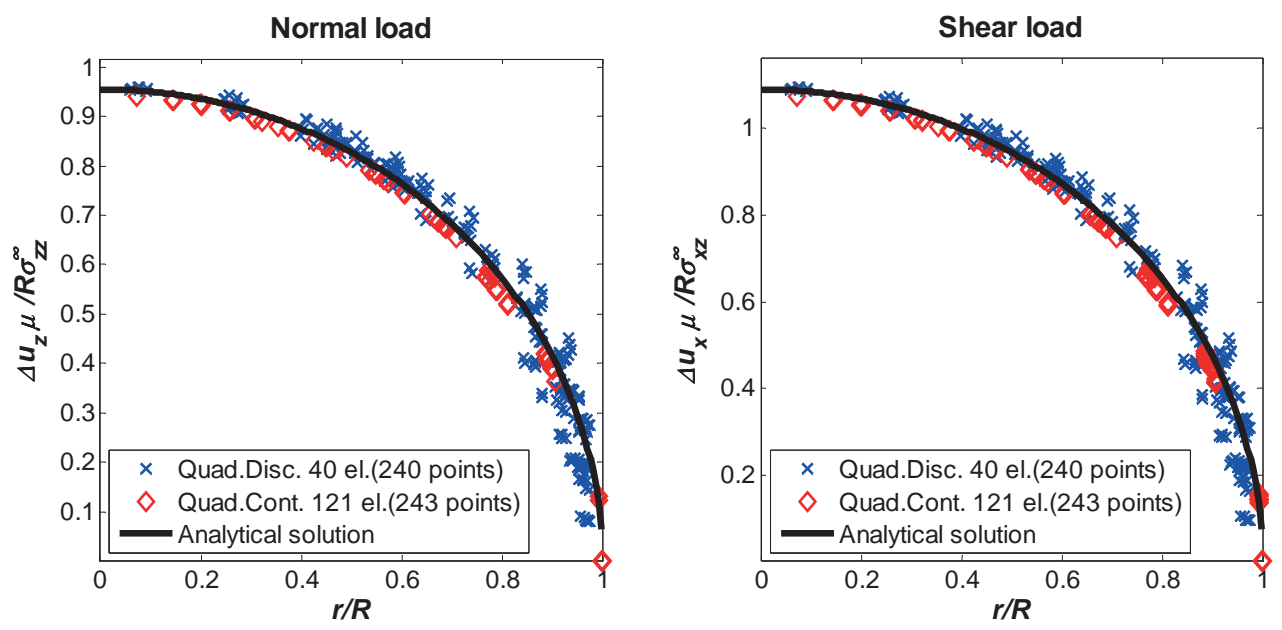

Figure 3: Normalized displacement discontinuity $(\Delta \mathbf{u} / R S)$ for a penny-shaped crack under normal loading with $S=\sigma_{z z}^{\infty} / \mu$ (left) and shear loading with $S=\sigma_{x z}^{\infty} / \mu$ (right). Continuous (scheme a) and discontinuous (scheme b) quadratic approximations.
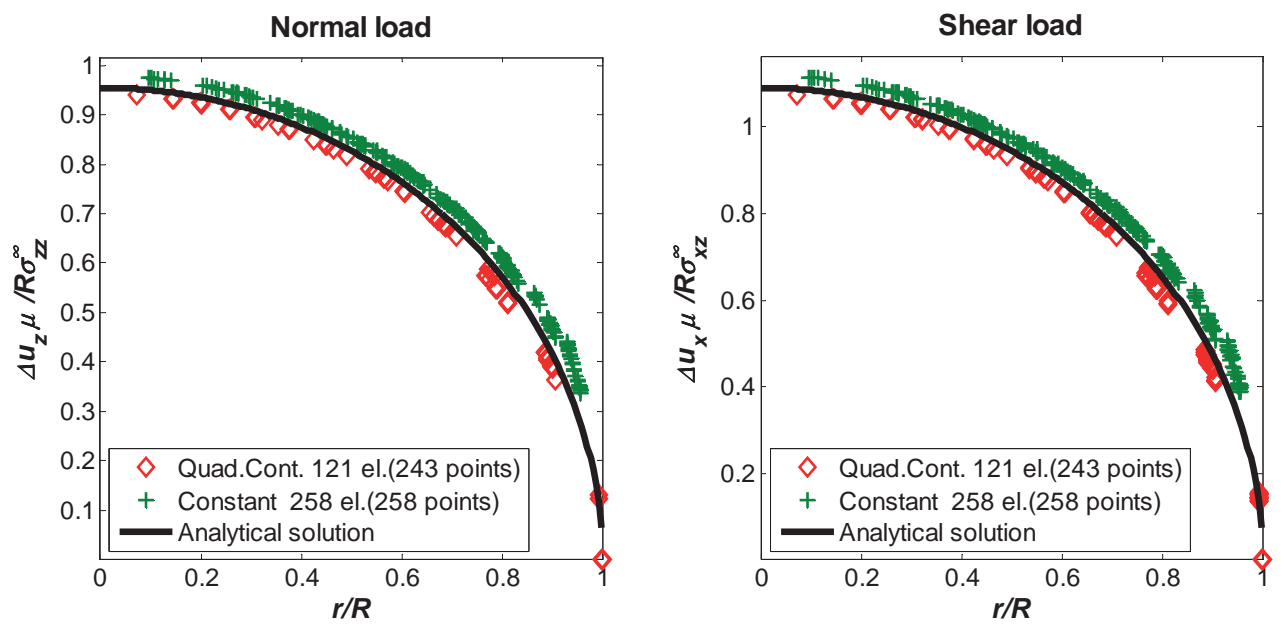

Figure 4: Normalized displacement discontinuity $(\Delta \mathbf{u} / R S)$ for a penny-shaped crack under normal loading with $S=\sigma_{z z}^{\infty} / \mu$ (left) and shear loading with $S=\sigma_{x z}^{\infty} / \mu$ (right). Continuous (scheme a) and constant approximations. 

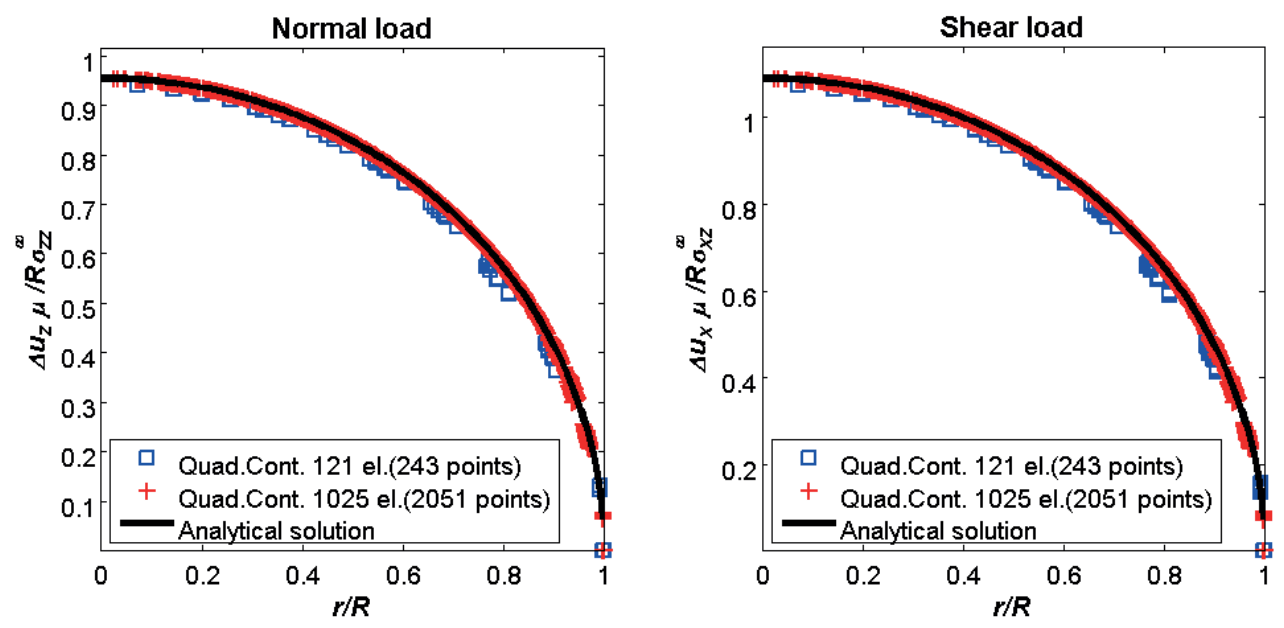

Figure 5: Normalized displacement discontinuity $(\Delta \mathbf{u} / R S)$ for a penny-shaped crack under normal loading with $S=\sigma_{z z}^{\infty} / \mu$ (left) and shear loading with $S=\sigma_{x z}^{\infty} / \mu$ (right). Quadratic approximations (scheme a) on meshes with 121 and 1025 elements.

To compare the stresses near the crack tip we evaluated them on the line AB shown in Fig. 6. The results (dimensionless stresses $\sigma_{z z} / \sigma_{z z}^{\infty}$ for normal load and $\sigma_{j k} / \sigma_{x z}^{\infty}$ for shear load vs. dimensionless distance from the crack tip $d / R$ ) presented in Figs. 7-8 are calculated on the lines characterized by different angles $\alpha, \beta$ using quadratic and constant approximation schemes. The dimensionless stresses along the line with $\alpha=\beta=45^{\circ}$ are also tabulated in Table 1 along with the corresponding analytical solutions (see [8],[14]-[17]). It could be seen from these figures and the table that quadratic approximations somehow reflect the asymptotic behavior of stresses, while constant approximations do not. The accuracy of the tabulated solution, except for the region very close to the crack tip (less than 1/10 the size of an element), as well as near the points where the stresses are close to zero, is within 10\% for quadratic approximations (121 elements) and up to $64 \%$ for constant approximations (258 elements). Note again that we did not employ any special crack tip shape functions in our method.

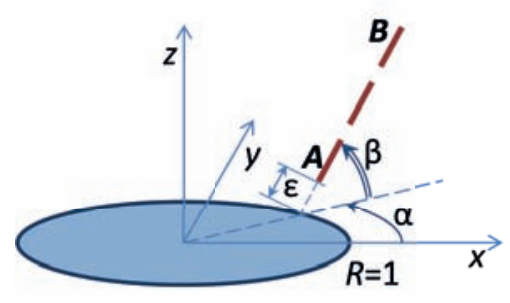

Figure 6: Penny-shaped crack. Location of points for evaluation of stresses near the crack tip. 

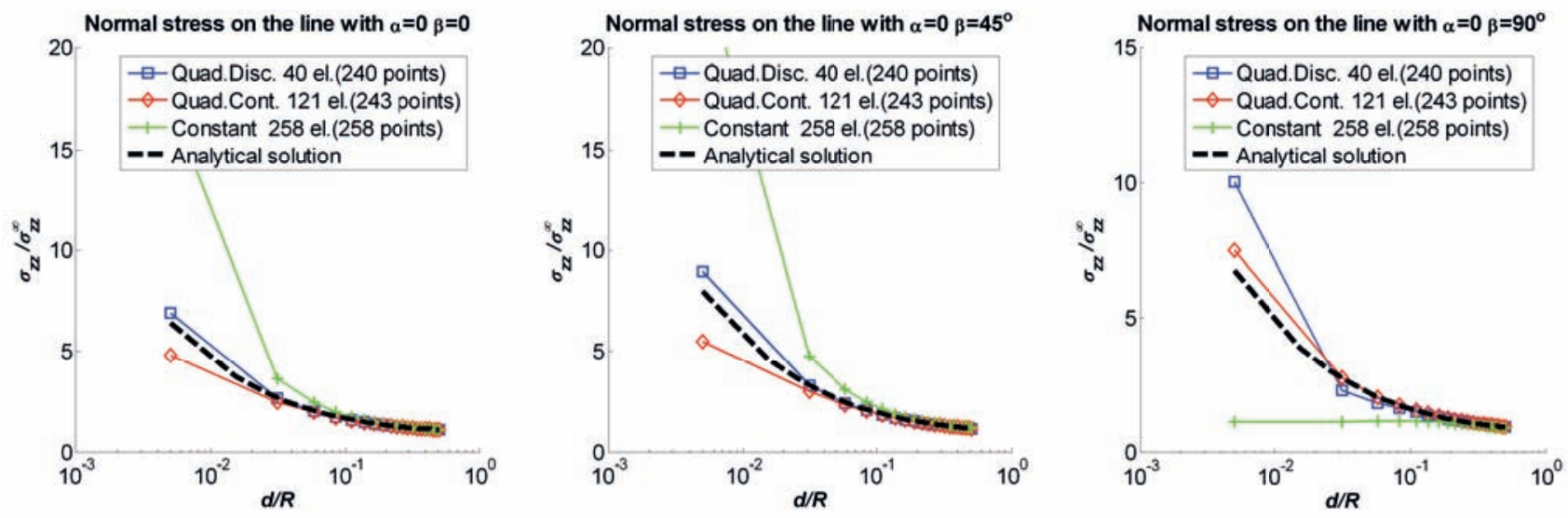

Figure 7: Dimensionless normal stresses $\left(\sigma_{z z} / \sigma_{z z}^{\infty}\right)$ near the tip of a penny shape crack under normal load.
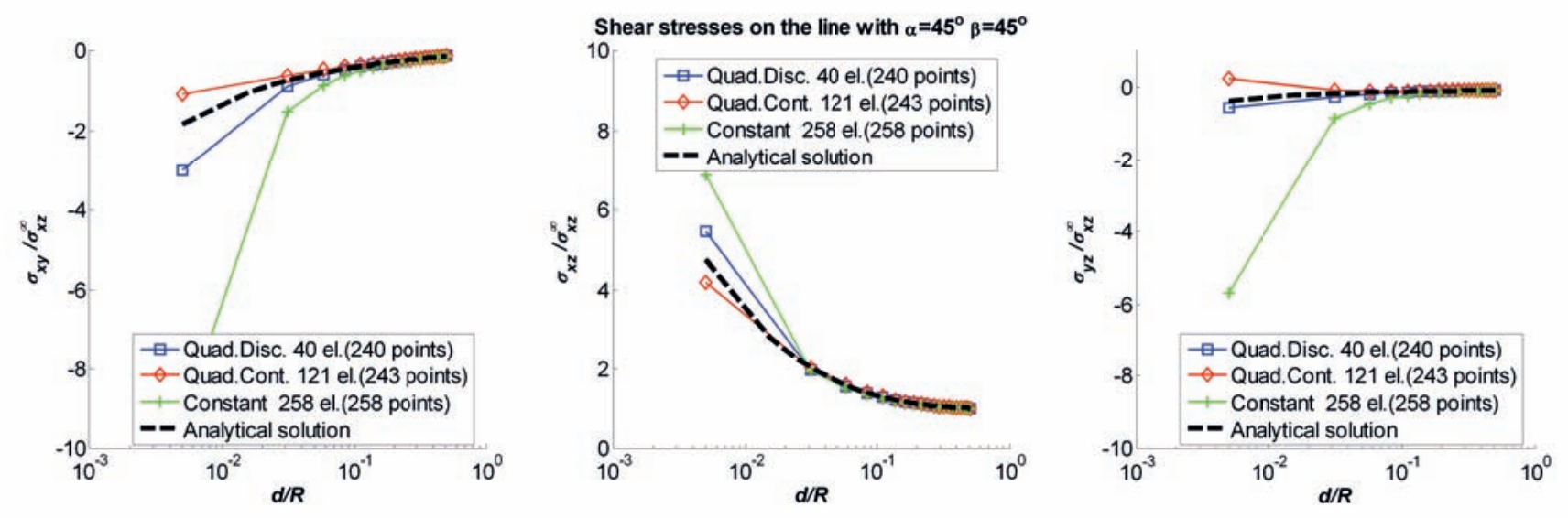

Figure 8: Dimensionless shear stresses $\left(\sigma_{j k} / \sigma_{x z}^{\infty}\right)$ near the tip of a penny shape crack under shear load. 
Table 1: Penny-shaped crack under normal $\left(S=\sigma_{z z}^{\infty} / \mu\right)$ and shear $\left(S=\sigma_{x z}^{\infty} / \mu\right)$ load. Dimensionless stresses $\left(\widetilde{\sigma}_{j k}=\sigma_{j k} / \mu S\right)$ at points near the crack tip (line AB with $\left.\alpha=\beta=45^{\circ}\right)$.

\begin{tabular}{|c|c|c|c|c|c|c|c|c|c|c|c|}
\hline \multicolumn{12}{|c|}{ Analytical solution } \\
\hline \multirow{2}{*}{$d$} & \multirow{2}{*}{$x=y$} & \multirow{2}{*}{$z$} & \multicolumn{6}{|c|}{ Normal load $\left(S=\sigma_{z z}^{\infty} / \mu\right)$} & \multicolumn{3}{|c|}{ Shear load $\left(S=\sigma_{x z}^{\infty} / \mu\right)$} \\
\hline & & & \multicolumn{2}{|c|}{$\widetilde{\sigma}_{x x}=\widetilde{\sigma}_{y y}$} & $\widetilde{\sigma}_{x y}$ & \multicolumn{2}{|c|}{$\widetilde{\sigma}_{x z}=\widetilde{\sigma}_{y z}$} & $\widetilde{\sigma}_{z z}$ & $\widetilde{\sigma}_{x y}$ & $\widetilde{\sigma}_{x z}$ & $\widetilde{\sigma}_{y z}$ \\
\hline 0.005 & 0.7096 & 0.0035 & \multicolumn{2}{|c|}{2.6699} & 0.4135 & \multicolumn{2}{|c|}{0.5989} & 8.0034 & -1.8236 & 4.7566 & -0.3589 \\
\hline 0.205 & 0.8096 & 0.1450 & \multicolumn{2}{|c|}{0.0636} & -0.0074 & \multicolumn{2}{|c|}{0.0451} & 1.5024 & -0.2570 & 1.1087 & -0.0959 \\
\hline 0.405 & 0.9096 & 0.2864 & \multicolumn{2}{|c|}{0.0012} & -0.0266 & \multicolumn{2}{|c|}{0.0123} & 1.2344 & -0.1530 & 1.0149 & -0.0742 \\
\hline 0.605 & 1.0096 & 0.4278 & \multicolumn{2}{|c|}{-0.0111} & -0.0281 & \multicolumn{2}{|c|}{0.0008} & 1.1360 & -0.1022 & 0.9938 & -0.0582 \\
\hline 0.805 & 1.1096 & 0.5692 & \multicolumn{2}{|c|}{-0.0130} & -0.0253 & \multicolumn{2}{|c|}{-0.0039} & 1.0873 & -0.0720 & 0.9886 & -0.0459 \\
\hline 1.005 & 1.2096 & 0.7106 & \multicolumn{2}{|c|}{-0.0122} & -0.0218 & \multicolumn{2}{|c|}{-0.0056} & 1.0595 & -0.0525 & 0.9881 & -0.0366 \\
\hline \multicolumn{12}{|c|}{ Constant approximations (258 elements) } \\
\hline \multirow{2}{*}{$d$} & \multirow{2}{*}{$x=y$} & \multirow{2}{*}{$z$} & \multicolumn{6}{|c|}{ Normal load $\left(S=\sigma_{z z}^{\infty} / \mu\right)$} & \multicolumn{3}{|c|}{ Shear load $\left(S=\sigma_{x z}^{\infty} / \mu\right)$} \\
\hline & & & $\widetilde{\sigma}_{x x}$ & $\widetilde{\sigma}_{y y}$ & $\widetilde{\sigma}_{x y}$ & $\widetilde{\sigma}_{x z}$ & $\widetilde{\sigma}_{y z}$ & $\widetilde{\sigma}_{z z}$ & $\widetilde{\sigma}_{x y}$ & $\widetilde{\sigma}_{x z}$ & $\widetilde{\sigma}_{y z}$ \\
\hline 0.005 & 0.7096 & 0.0035 & 3.4939 & 3.5542 & -2.5778 & 0.3943 & 0.3863 & 26.2161 & -9.2875 & 6.8749 & -5.6704 \\
\hline 0.205 & 0.8096 & 0.1450 & 0.0411 & 0.0453 & -0.0379 & 0.0226 & 0.0228 & 1.5868 & -0.2958 & 1.0851 & -0.1389 \\
\hline 0.405 & 0.9096 & 0.2864 & -0.0063 & -0.0043 & -0.0364 & 0.0044 & 0.0051 & 1.2584 & -0.1672 & 1.0069 & -0.0885 \\
\hline 0.605 & 1.0096 & 0.4278 & -0.0147 & -0.0141 & -0.0331 & -0.0031 & -0.0028 & 1.1473 & -0.1100 & 0.9899 & -0.0657 \\
\hline 0.805 & 1.1096 & 0.5692 & -0.0152 & -0.0150 & -0.0285 & -0.0061 & -0.0060 & 1.0938 & -0.0771 & 0.9863 & -0.0507 \\
\hline 1.005 & 1.2096 & 0.7106 & -0.0137 & -0.0136 & -0.0240 & -0.0072 & -0.0071 & 1.0637 & -0.0561 & 0.9865 & -0.0399 \\
\hline \multicolumn{12}{|c|}{ Quadratic approximations (121 elements) } \\
\hline \multirow{2}{*}{$d$} & \multirow{2}{*}{$x=y$} & \multirow{2}{*}{$z$} & & & nal loac & $\left(S=\sigma_{z z}^{\infty}\right.$ & & & Shear l & $\operatorname{ad}(S=$ & $\left.\sigma_{x z}^{\infty} / \mu\right)$ \\
\hline & & & $\widetilde{\sigma}_{x x}$ & $\widetilde{\sigma}_{y y}$ & $\widetilde{\sigma}_{x y}$ & $\widetilde{\sigma}_{x z}$ & $\widetilde{\sigma}_{y z}$ & $\widetilde{\sigma}_{z z}$ & $\widetilde{\sigma}_{x y}$ & $\widetilde{\sigma}_{x z}$ & $\widetilde{\sigma}_{y z}$ \\
\hline 0.005 & 0.7096 & 0.0035 & 2.6828 & 2.9155 & 0.8992 & 0.6430 & 0.6706 & 6.1098 & -1.1884 & 4.7212 & 0.2779 \\
\hline 0.205 & 0.8096 & 0.1450 & 0.0660 & 0.0646 & -0.0100 & 0.0493 & 0.0488 & 1.5292 & -0.2670 & 1.1147 & -0.1019 \\
\hline 0.405 & 0.9096 & 0.2864 & 0.0001 & $\begin{array}{l}-0.0000 \\
\end{array}$ & -0.0291 & 0.0120 & 0.0121 & 1.2449 & -0.1581 & 1.0147 & -0.0779 \\
\hline 0.605 & 1.0096 & 0.4278 & -0.0121 & -0.0122 & $\begin{array}{l}-0.0298 \\
\end{array}$ & 0.0002 & 0.0002 & 1.1413 & -0.1054 & 0.9932 & $\begin{array}{l}-0.0606 \\
\end{array}$ \\
\hline 0.805 & 1.1096 & 0.5692 & -0.0138 & -0.0138 & -0.0266 & -0.0044 & $\begin{array}{l}-0.0044 \\
-0.04\end{array}$ & 1.0904 & -0.0742 & 0.9880 & -0.0476 \\
\hline 1.005 & 1.2096 & 0.7106 & -0.0127 & -0.0128 & -0.0227 & -0.0061 & -0.0061 & 1.0616 & -0.0541 & 0.9876 & -0.0378 \\
\hline
\end{tabular}

\section{Benchmark 2: Two semi-cylindrical coaxial cracks}

Consider two coaxial cracks of cylindrical shape of the same radius (Fig. 9a) under biaxial far-field tension perpendicular to the axis. Assume that the length in the axial direction is much longer than the curvature radius. Thus, it is reasonable to expect that the fields in the middle cross-section of such system would mimic the solution of a 2D problem of two cracks along the same circle (Fig. 9b). The corresponding 2D problem can be solved analytically [18] or numerically [19]. 


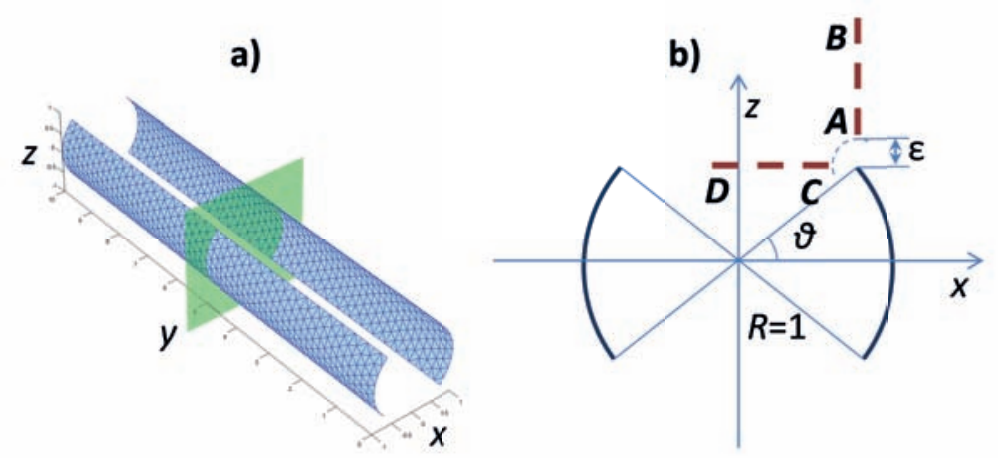

Figure 9: Two coaxial cylindrical cracks under biaxial tension a) and their central cross section b). Location of points for evaluation of stresses near the crack tip.

The three-dimensional problem was solved for the following parameters: curvature radius $R=1$, aspect ratio (length vs. curvature radius) $L / R=10$, aperture angle $\vartheta=45^{\circ}$. The dimensionless far-field stresses are set as follows: $S=\sigma_{x x}^{\infty} / \mu=\sigma_{z z}^{\infty} / \mu$. The results (dimensionless stress components in $x-z$ plane) are calculated on two straight lines in the central cross section starting at one of the crack tips (lines AB and CD shown in Fig. $9 \mathrm{~b})$. As in the previous Figures, $d$ is the distance from the crack tip. Figs. 10-11 compares the three-dimensional results calculated using quadratic and constant approximations to the two-dimensional results obtained using the algorithm described in [19] with circular elements. Note that this algorithm employed special square root crack tip elements while the three-dimensional algorithm did not.

Table 2 presents the dimensionless stresses calculated at some points near one of the crack tips (on the lines $\mathrm{AB}$ and $\mathrm{CD}$ in Fig. 9b). Table 3 presents the dimensionless stresses on the axial line of the cylinder $(x=z=0)$. The latter stresses are also plotted in Fig. $12\left(\sigma_{x z} / \mu S\right.$ is not shown as it was equal to zero within the 0.005 accuracy).

The graphic and tabulated results confirm that the two-dimensional plane strain model provides reasonable estimates of the stresses in the middle cross-section.
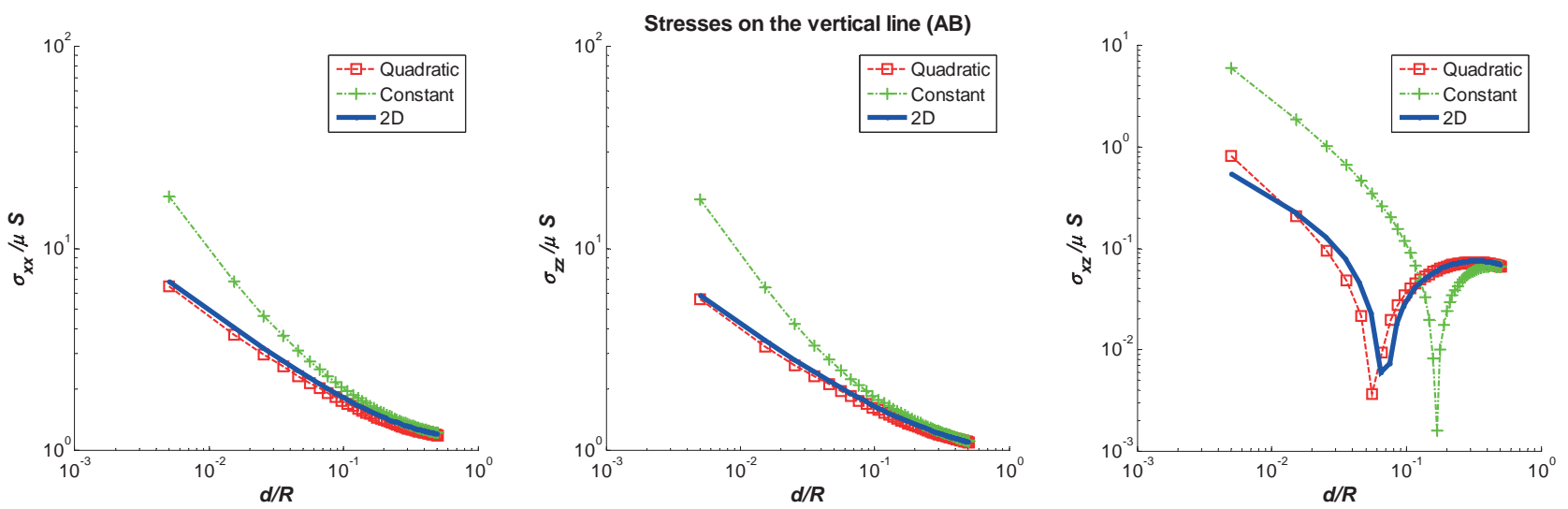

Figure 10: Two coaxial cylindrical cracks under biaxial tension. Dimensionless stresses near the crack tip (on the line $\mathrm{AB}$ in Fig. 9b). 

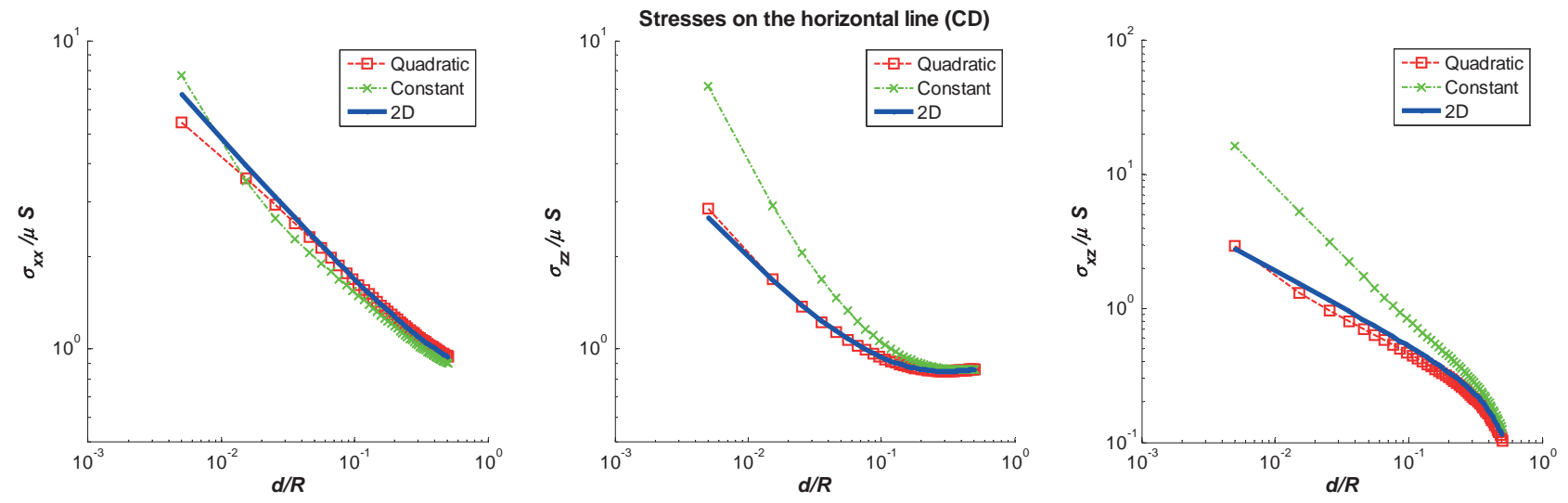

Figure 11: Two coaxial cylindrical cracks under biaxial tension. Dimensionless stresses near the crack tip (on the line CD in Fig. 9b).

Table 2: Two cylindrical cracks under biaxial tension $\left(S=\sigma_{x x}^{\infty} / \mu=\sigma_{z z}^{\infty} / \mu\right)$. Dimensionless stresses $\left(\widetilde{\sigma}_{j k}=\right.$ $\sigma_{j k} / \mu S$ ) in the central cross-section near the crack tip (on the lines AB and CD in Fig. 9b).

\begin{tabular}{|c|c|c||c|c|c||c|c|c|}
\hline \multirow{2}{*}{$d$} & \multirow{2}{*}{$x$} & \multirow{2}{*}{$z$} & \multicolumn{3}{|c||}{ 2D solution } & \multicolumn{3}{|c|}{ 3D BEM solution } \\
\cline { 5 - 9 } & & & $\widetilde{\sigma}_{x x}$ & $\widetilde{\sigma}_{z z}$ & $\widetilde{\sigma}_{x z}$ & $\widetilde{\sigma}_{x x}$ & $\widetilde{\sigma}_{z z}$ & $\widetilde{\sigma}_{x z}$ \\
\hline \hline 0.005 & 0.7071 & 0.7121 & 6.8466 & 5.8725 & 0.5510 & 6.4854 & 5.5940 & 0.8229 \\
\hline 0.105 & 0.7071 & 0.8121 & 1.7864 & 1.6298 & -0.0338 & 1.7166 & 1.5902 & -0.0391 \\
\hline 0.205 & 0.7071 & 0.9121 & 1.4436 & 1.3356 & -0.0676 & 1.4044 & 1.3150 & -0.0667 \\
\hline 0.305 & 0.7071 & 1.0121 & 1.3118 & 1.2127 & -0.0751 & 1.2834 & 1.1997 & -0.0727 \\
\hline 0.405 & 0.7071 & 1.1121 & 1.2449 & 1.1444 & -0.0739 & 1.2216 & 1.1353 & -0.0709 \\
\hline 0.505 & 0.7071 & 1.2121 & 1.2057 & 1.1015 & -0.0687 & 1.1853 & 1.0948 & -0.0656 \\
\hline \hline 0.005 & 0.7021 & 0.7071 & 6.7404 & 2.6827 & 2.7857 & 5.4604 & 2.8641 & 2.9524 \\
\hline 0.105 & 0.6021 & 0.7071 & 1.6536 & 0.9347 & 0.5080 & 1.6320 & 0.9310 & 0.4496 \\
\hline 0.205 & 0.5021 & 0.7071 & 1.2620 & 0.8593 & 0.3281 & 1.2567 & 0.8590 & 0.2982 \\
\hline 0.305 & 0.4021 & 0.7071 & 1.0927 & 0.8481 & 0.2356 & 1.0922 & 0.8489 & 0.2174 \\
\hline 0.405 & 0.3021 & 0.7071 & 0.9992 & 0.8524 & 0.1677 & 1.0001 & 0.8536 & 0.1563 \\
\hline 0.505 & 0.2021 & 0.7071 & 0.9445 & 0.8598 & 0.1091 & 0.9459 & 0.8612 & 0.1023 \\
\hline
\end{tabular}



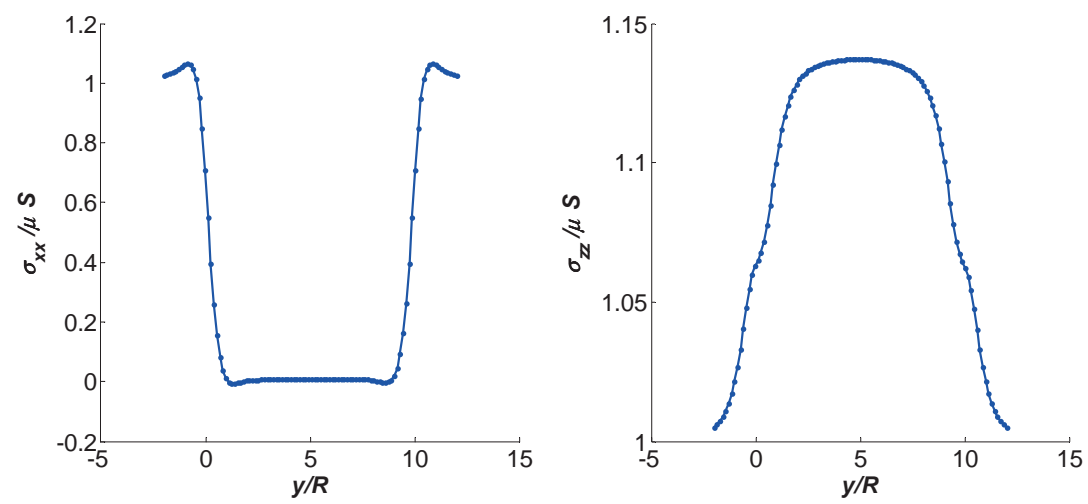

Figure 12: Two coaxial cylindrical cracks under biaxial tension. Dimensionless stresses $\left(\sigma_{x x} / \mu S, \sigma_{z z} / \mu S\right)$ along the axial line.

Table 3: Dimensionless stresses $\left(\widetilde{\sigma}_{j k}=\sigma_{j k} / \mu S\right)$ on the axis of two cylindrical cracks under biaxial tension $\left(S=\sigma_{x x}^{\infty} / \mu=\sigma_{z z}^{\infty} / \mu\right)$.

\begin{tabular}{|c||c|c|c|}
\hline$y$ & $\widetilde{\sigma}_{x x}$ & $\widetilde{\sigma}_{y y}$ & $\widetilde{\sigma}_{z z}$ \\
\hline \hline $2 \mathrm{D}$ & 0.0000 & - & 1.1458 \\
\hline \hline 5.0 & 0.0275 & -0.2120 & 1.1439 \\
\hline 6.0 & 0.0275 & -0.2106 & 1.1433 \\
\hline 7.0 & 0.0272 & -0.2067 & 1.1413 \\
\hline 8.0 & 0.0227 & -0.1921 & 1.1348 \\
\hline 9.0 & 0.0378 & -0.1103 & 1.1078 \\
\hline 10.0 & 0.7162 & -0.1309 & 1.0627 \\
\hline 11.0 & 1.0582 & -0.0480 & 1.0202 \\
\hline 12.0 & 1.0224 & 0.0031 & 1.0047 \\
\hline
\end{tabular}

\section{Concluding remarks}

A new boundary element method technique with quadratic approximations is suggested for the analysis of multiple three-dimensional cracks of arbitrary shapes. The technique employs complex variables for representations of the elastic fields and triangular elements for boundary approximations. The use of Cauchy-Pompeiu theorem allows for the efficient analytical treatment of all element integrals involved in the representations of the fields. The boundary conditions are enforced using the "limit after discretization" procedure that produces no singular integrals; the same procedure is used to set up the system of linear algebraic equations for the boundary unknowns and to evaluate the elastic fields everywhere in the domain of interest.

Two approximations schemes that involve quadratic polynomials are employed and compared. Using two benchmark examples (the problem of a penny-shaped crack and the problem of two coaxial cracks of cylindrical shape), it is demonstrated that continuous quadratic approximations provide better results than its discontinuous counterpart. We believe that it is so due to the fact that, in the first scheme, the conditions of zero displacement discontinuity were enforced at the nodal points located at the crack front. The results, both tabulated and presented in graphical form, indicate that the continuous quadratic approximation scheme yields much better accuracy than constant approximation scheme for roughly the same numbers of degrees of freedom. In addition, quadratic approximations allow for better capturing asymptotic behavior of stresses near crack fronts than constant approximations. Some of the tabulated results presented here can be used as benchmarks for future 
investigations.

In the present work, square root crack tip elements have not been employed as our method could be used e.g. in application to hydraulic fracturing, in which the asymptotic of crack opening displacement is governed by a specific propagation regime and may not necessarily be of square root type. However, we believe that the use of Cauchy-Pompeiu theorem may provide analytical results for the special shape elements as well. We intend to explore it in the future work, which would also include issues related to efficient solution of the linear algebraic equations using fast methods.

\section{Acknowledgements}

Partial support was provided by DOE Grant DE-FE0002020 funded through the American Recovery and Reinvestment Act. The second author gratefully acknowledges the support from the Theodore W. Bennett Chair, University of Minnesota.

\section{References}

[1] Crouch SL, Starfield AM. Boundary element methods in solid mechanics. London: Allen and Unwin; 1983.

[2] Aliabadi MH. Boundary element method: Applications to solids and structures, vol. 2. Chicester, England: John Wiley \& Sons, Ltd.; 2002.

[3] Adachi J, Siebrits E, Peirce A, Desroches J. Computer simulation of hydraulic fractures. International Journal of Rock Mechanics and Mining Sciences 2007; 44: 739-757

[4] Nikolskiy DV, Mogilevskaya SG, Labuz JF. Complex variables boundary element analysis of threedimensional crack problems. Engineering Analysis with Boundary Elements 2013; 37: 1532-1544

[5] Barber JR, Solid mechanics and its applications: Elasticity. London, New York: Springer Science; 2010.

[6] Piltner R, The use of complex valued functions for the solution of three-dimensional elasticity problems. Journal of Elasticity 1987; 18: 191-225

[7] Piltner R, On the representation of three-dimensional elasticity solutions with the aid of complex valued functions. Journal of Elasticity 1989; 22: 45-55

[8] Fabrikant VI. Penny-shaped crack revisited: closed-form solutions. Philosophical Magazine A. 1987; 56 (2): 191-207

[9] Mogilevskaya SG. Lost in translation: Crack problems in different languages. International Journal of Solids and Structures 2014. DOI: 10.1016/j.ijsolstr.2014.08.025

[10] Mogilevskaya SG, Nikolskiy DV. The use of complex integral representations for analytical evaluation of three-dimensional BEM integrals. Potential and Elasticity problems. The Quarterly Journal of Mechanics and Applied Mathematics 2014; 67: 505-523

[11] Kupradze VD. Potential Methods in the Theory of Elasticity. Jerusalem: Israel Program for Scientific Translations; 1965. (Translation of Russian edition. 1963. Gos. Izdat. Fiz-Mat Lit, Moscow).

[12] Parton VZ, Perlin PI. Integral Equations in Elasticity. Moscow: Mir Publishers; 1982.

[13] Linkov AM. Boundary Integral Equations in Elasticity Theory. Dordrecht, Netherlands: Kluwer Academic Publishers; 2002.

[14] Sack RA. Extension of Griffith's theory of rupture to three dimensions. Proceedings of the Physical Society London 1946; 58: 729 
[15] Sneddon IN. The distribution of stress in the neighbourhood of a crack in an elastic solid. Proceedings of the Royal Society A. 1946: 187-229

[16] Green AE. On Boussinesq's problem and penny-shaped cracks. Mathematical Proceedings of the Cambridge Philosophical Society 1949; 45 (2): 251-257

[17] Segedin CM. Note on a penny-shaped crack under shear. Mathematical Proceedings of the Cambridge Philosophical Society 1951; 47 (2): 396-400

[18] Muskhelishvili NI. Some Basic Problems of the Mathematical Theory of Elasticity - fundamental equations, plane theory of elasticity, torsion, and bending. Groningen: P.Noordhoff; 1963.

[19] Mogilevskaya SG. The universal algorithm based on complex hypersingular integral equation to solve plane elasticity problems. Computational Mechanics 1996; 18: 127-138

[20] Vekua IN. Generalized analytic functions. Oxford: Pergamon Press; 1962. 


\section{Appendix A. Evaluation of the generic integral}

Area integral (15) can be reduced to a contour integral with the use of Cauchy-Pompeiu (Borel-Pompeiu) representation formula (see [20]):

$$
\frac{1}{2 \pi i} \int_{\partial \Sigma} \frac{f(\tau) d \tau}{\tau-z}-\frac{1}{\pi} \int_{\Sigma} \frac{\partial f(\tau)}{\partial \bar{\tau}} \frac{d \Sigma}{\tau-z}=\left\{\begin{array}{cc}
f(z) & \mathbf{z} \in \Sigma \\
0 & \mathbf{z} \notin \Sigma \cup \partial \Sigma
\end{array}\right.
$$

where $f$ is a sufficiently smooth function and $\Sigma$ is a region on the complex plane bounded by a simple (non-selfintersecting) curve $\partial \Sigma$.

The limit values (when the point $\mathbf{z} \in \Sigma$ approaches the point $\mathbf{z}_{\mathbf{0}} \in \partial \Sigma$ associated with the complex combination $z_{0}=z_{10}+i z_{20}$ ) of Cauchy type integrals involved in (20) can be evaluated using Sokhotsli-Plemelj formulae (see [18]) as follows:

$$
\lim _{\mathbf{z} \rightarrow \mathbf{z}_{0}} \frac{1}{2 \pi i} \int_{\partial \Sigma} \frac{f(\tau) d \tau}{\tau-z}=\frac{\gamma}{2 \pi} f\left(z_{0}\right)+\frac{1}{2 \pi i} f \frac{f(\tau) d \tau}{\partial \Sigma}
$$

in which $f$ is understood as a Cauchy principal value integral, and $\gamma$ is an internal angle of the curve $\partial \Sigma$ at the point $\mathbf{z}_{0}$.

Thus, for integral (15) on the triangular element $E_{s}$, we have

$$
I_{m n}=\int_{E_{s}} \frac{(\tau-z)^{m}(\bar{\tau}-\bar{z})^{n}}{r} d \Sigma=\frac{1}{2 i} \int_{\partial E_{s}} \frac{f(\tau) d \tau}{\tau-z}-\left\{\begin{array}{cc}
\pi f(z) & \mathbf{z} \in E_{s} \\
\frac{\gamma}{2} f(z) & \mathbf{z} \in \partial E_{s} \\
0 & \mathbf{z} \notin E_{s}
\end{array}\right.
$$

where $f(\tau)$ is given by the following closed form expression:

$$
f(\tau)=\left\{\begin{array}{cc}
2 r(\tau-z)^{m} & n=0 \\
\frac{2}{3} r\left(r^{2}-3 h^{2}\right)(\tau-z)^{m-1} & n=1 \\
\frac{2}{15} r\left(3 r^{4}-10 h^{2} r^{2}+15 h^{4}\right)(\tau-z)^{m-2} & n=2
\end{array}\right.
$$

The countour integral involved in Eq.(22) is path-dependent, as the function $\frac{f(\tau)}{\tau-z}$, in general, is not holomorphic. For triangular element, $\partial E_{s}=\bigcup_{m=1}^{3}\left[\mathbf{a}_{m}, \mathbf{b}_{m}\right], \mathbf{b}_{m}=\mathbf{a}_{m+1}, \mathbf{b}_{3}=\mathbf{a}_{1}, a_{m}=a_{1 m}+i a_{2 m}, b_{m}=b_{1 m}+i b_{2 m}$, and the contour integral can be evaluated as a sum of integrals over the three sides of the triangle. For each side (straight segment with the beginning point $\mathbf{a}_{m}$ and the end point $\mathbf{b}_{m}$ ), the variable $\tau$ and its conjugate are related through the following equation (that defines a specific integration path):

$$
\bar{\tau}=\bar{a}_{m}+\frac{\bar{b}_{m}-\bar{a}_{m}}{b_{m}-a_{m}}\left(\tau-a_{m}\right)
$$

Thus, the distance $r$ can be expressed via the following rational function of $\tau$ :

$$
r(\tau)=\sqrt{(\tau-z)\left[D_{m}(\tau-z)+2 \bar{d}_{m}\right]+h^{2}}
$$

where $D_{m}=\left(\bar{b}_{m}-\bar{a}_{m}\right) /\left(b_{m}-a_{m}\right)$ and $d_{m}=d_{1 m}+i d_{2 m}=\frac{1}{2}\left[a_{m}-z-\bar{D}_{m}\left(\bar{a}_{m}-\bar{z}\right)\right]$. Note that the latter has the meaning of complex combination of the components of the vector originating at the point $\mathbf{z}$ and perpendicular to the contour segment $\left[\mathbf{a}_{m}, \mathbf{b}_{m}\right]$ (see Fig. 2). Also note that $\left|d_{m}\right|=0$ if $\mathbf{z} \in\left(\mathbf{a}_{m}, \mathbf{b}_{m}\right)$, and $D_{m}=-\bar{d}_{m} / d_{m}$ if $\mathbf{z} \notin\left[\mathbf{a}_{m}, \mathbf{b}_{m}\right]$. 


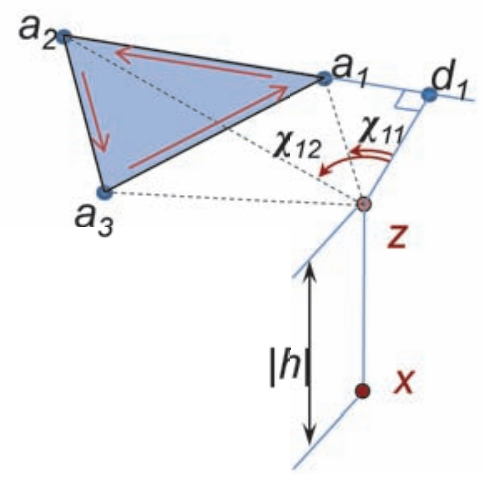

Figure 13: Boundary element. Integration scheme and notations.

With the above notations, expressions for the function $f(\tau)$ of (23) can be rewritten as follows:

$$
f(\tau)=\left\{\begin{array}{cc}
2 r(\tau)(\tau-z)^{m} & n=0 \\
\frac{2}{3} r(\tau)\left[D_{a b}(\tau-z)^{m+1}+2 \bar{d}_{m}(\tau-z)^{m}-2 h^{2}(\tau-z)^{m-1}\right] & n=1 \\
\frac{2}{15} r(\tau)\left[3 D_{m}^{2}(\tau-z)^{m+2}+12 \bar{d}_{m} D_{m}(\tau-z)^{m+1}\right. & \\
\left.-4 D_{m}\left(3\left|d_{m}\right|^{2}+h^{2}\right)(\tau-z)^{m}-8 \bar{d}_{m} h^{2}(\tau-z)^{m-1}+8 h^{4}(\tau-z)^{m-2}\right] & n=2
\end{array}\right.
$$

Substitution of expressions (26) into (22) results, for all cases of $n$, in the integrals of the same following type:

$$
J_{k, m}(z)=\int_{a_{m}}^{b_{m}} r(\tau)(\tau-z)^{k} d \tau
$$

with $-1 \leq k \leq 4$. The results of analytical integration of (27) are reported in [10]. Here we provide final closed-form expressions for the total integral over the contour $\partial E_{s}$ :

$$
J_{k}=\int_{\partial E_{s}} r(\tau)(\tau-z)^{k} d \tau=\sum_{m=1}^{3}\left\{\begin{array}{cc}
{\left.\left[-\left|d_{m}\right| L_{0}-|h| G_{0}\right]\right|_{\tau=a_{m}} ^{\tau=a_{m}}} & k=-1 \\
\left.\frac{1}{2}\left[r d_{m}+C_{m}\left(\left|d_{m}\right|^{2}+h^{2}\right) L_{0}\right]\right|_{\tau=a_{m}} ^{\tau=b_{m}} & k=0 \\
\frac{\bar{D}_{m}}{k+2}\left[\begin{array}{c}
\left.r^{3}(\tau-z)^{k-1}\right|_{\tau=b_{m}} ^{\tau=b_{m}}-\bar{d}_{m}(2 k+1) J_{k-1, m}(z) \\
\left.-h^{2}(k-1) J_{k-2, m}(z)\right]
\end{array}\right. & k \geq 1
\end{array}\right.
$$

where $C_{m}=\frac{b_{m}-a_{m}}{\left|b_{m}-a_{m}\right|}$ and $L_{0}, G_{0}$ are given by the following expressions:

$$
\begin{aligned}
L_{0} & = \begin{cases}\tan ^{-1} \frac{\left|d_{m}\right|\left(d_{m}-\tau+z\right)}{d r} & \mathbf{z} \notin\left[\mathbf{a}_{m}, \mathbf{b}_{m}\right] \\
\sinh ^{-1} \frac{\tau-z}{|h|} & \mathbf{z} \in\left(\mathbf{a}_{m}, \mathbf{b}_{m}\right)\end{cases} \\
G_{0} & = \begin{cases}\tanh ^{-1} \frac{|h|\left(d_{m}-\tau+z\right)}{d_{m} r} & \mathbf{z} \notin\left[\mathbf{a}_{m}, \mathbf{b}_{m}\right] \\
\ln \frac{r+|h|}{|\tau-z|} & \mathbf{z} \in\left(\mathbf{a}_{m}, \mathbf{b}_{m}\right)\end{cases}
\end{aligned}
$$

The integral involved in (28) for $k=-1$ and $\mathbf{z} \in\left(\mathbf{a}_{m}, \mathbf{b}_{m}\right)$ is understood as Cauchy principal value integral.

The integrals (28) should be assembled using (26) and substituted into (22) to get the final closed-form expression of the integral (15). 


\section{Figure captions}

Figure 1. Boundary element. Local coordinate system and notations.

Figure 2. Example of the boundary mesh (penny-shaped crack) and two schemes of nodes arrangement: a) continuous DD b) discontinuous DD.

Figure 3. Normalized displacement discontinuity $(\Delta \mathbf{u} / R S)$ for a penny-shaped crack under normal loading with $S=\sigma_{z z}^{\infty} / \mu$ (left) and shear loading with $S=\sigma_{x z}^{\infty} / \mu$ (right). Continuous (scheme a) and discontinuous (scheme b) quadratic approximations.

Figure 4. Normalized displacement discontinuity $(\Delta \mathbf{u} / R S)$ for a penny-shaped crack under normal loading with $S=\sigma_{z z}^{\infty} / \mu$ (left) and shear loading with $S=\sigma_{x z}^{\infty} / \mu$ (right). Continuous (scheme a) and constant approximations.

Figure 5. Normalized displacement discontinuity $(\Delta \mathbf{u} / R S)$ for a penny-shaped crack under normal loading with $S=\sigma_{z z}^{\infty} / \mu$ (left) and shear loading with $S=\sigma_{x z}^{\infty} / \mu$ (right). Quadratic approximations (scheme a) on meshes with 121 and 1032 elements.

Figure 6. Penny-shaped crack. Location of points for evaluation of stresses near the crack tip.

Figure 7. Dimensionless normal stress $\left(\sigma_{z z} / \sigma_{z z}^{\infty}\right)$ near the tip of a penny shape crack under normal load.

Figure 8. Dimensionless shear stresses $\left(\sigma_{j k} / \sigma_{x z}^{\infty}\right)$ near the tip of a penny shape crack under shear load.

Figure 9. Two coaxial cylindrical cracks under biaxial tension a) and their central cross section b). Location of points for evaluation of stresses near the crack tip.

Figure 10. Two coaxial cylindrical cracks under biaxial tension. Dimensionless stresses near the crack tip (on the line $\mathrm{AB}$ in Fig. 9b).

Figure 11. Two coaxial cylindrical cracks under biaxial tension. Dimensionless stresses near the crack tip (on the line CD in Fig. 9b).

Figure 12. Two coaxial cylindrical cracks under biaxial tension. Dimensionless stresses $\left(\sigma_{x x} / \mu, \sigma_{z z} / \mu\right)$ along the axial line.

Figure 13. Boundary element. Integration scheme and notations.

\section{Table headings}

Table 1. Penny-shaped crack under normal $\left(S=\sigma_{z z}^{\infty} / \mu\right)$ and shear $\left(S=\sigma_{x z}^{\infty} / \mu\right)$ load. Dimensionless stresses $\left(\widetilde{\sigma}_{j k}=\sigma_{j k} / \mu S\right)$ at points near the crack tip (line AB with $\left.\alpha=\beta=45^{\circ}\right)$.

Table 2. Two cylindrical cracks under biaxial tension $\left(S=\sigma_{x x}^{\infty} / \mu=\sigma_{z z}^{\infty} / \mu\right)$. Dimensionless stresses $\left(\widetilde{\sigma}_{j k}=\sigma_{j k} / \mu S\right)$ in the central cross-section near the crack tip (on the lines AB and CD in Fig. 9b).

Table 3. Dimensionless stresses $\left(\widetilde{\sigma}_{j k}=\sigma_{j k} / \mu S\right)$ on the axis of two cylindrical cracks under biaxial tension $\left(S=\sigma_{x x}^{\infty} / \mu=\sigma_{z z}^{\infty} / \mu\right)$. 